\title{
Fragmentable Heterogeneous Cocatalysts for the Metallocene-Catalyzed Polymerization of Olefins, I. Surface Modification of Silica and Characterization of the Resulting Carriers
}

\author{
Herbert Schumann, Ralf Widmaier, Katharina C. H. Lange, and Birgit C. Wassermann \\ Institut für Chemie, Technische Universität Berlin, Straße des 17. Juni 135, D-10623 Berlin, \\ Germany
}

Reprint requests to Prof. Dr. H. Schumann. E-mail: Schumann@chem.tu-berlin.de

Z. Naturforsch. 60b, $603-613$ (2005); received December 14, 2004

Surface modification of pre-dried spherical silica nano-particles with diameters of $235 \mathrm{~nm}$ and 10 to $20 \mathrm{~nm}$ and of commercially available non-spherical silica materials using various aminoalkyltrialkoxysilanes and $\alpha$ - $\omega$-bis(alkoxysilyl)organyls (organyl = alkanediyl, aminoalkanediyl, polyether, polysiloxane) has been performed in suspension in solvents with water. The quantity of water has a dominating influence, as compared to the amounts and the ratios of silanes employed, on the surface morphology of the modified silicas. The morphologies observed range from weakly linked aggregates of spherical particles to large agglomerates covered by thick irregular layers of organopolysiloxane, as demonstrated by scanning electron microscopy. These carriers can be modified further with organoaluminium compounds to yield heterogeneous cocatalysts for the polymerization of ethylene.

Key words: Silica, Surface Modification, Electron Microscopy, Silanes, Polymerization Cocatalysts

\section{Introduction}

Modified silica is used for various applications, including recently also catalysis [1]. In catalysis modified silicas are used to graft catalysts or cocatalysts onto silica in order to improve handling and catalytic stability, to change or direct the catalytic activity towards a desired product, or to perform heterogeneous reactions $[2 \mathrm{c}, \mathrm{e}]$. In the area of polymerization catalysis, active heterogeneous catalysts grafted via alkoxysilyl or other pendant groups require tailormade carriers exhibiting not only specific functional groups to graft the catalysts without decreasing or altering their activity, but which also fragment during polymerization as the polymers grow inside the particle [2].

Surface modification of $\mathrm{SiO}_{2}$ with organosilanes may be achieved employing different reaction types such as: 1. Condensation of silica surface silanol groups with silanes containing hydrolysable $\mathrm{Si}-\mathrm{X}$ groups $\left(\mathrm{X}=\mathrm{Cl}, \mathrm{Br}, \mathrm{I}, \mathrm{OH}, \mathrm{NR}_{2}, \mathrm{OR}, \mathrm{OCOR}\right.$ etc. $)$ $[1 \mathrm{~b}, 3,4] ; 2$. hydrosilylation of Si-H-modified silica with alkenyl- or alkinyl-functionalized compounds using Speier or Karstedt's catalyst [1b,5]; 3. SolGel synthesis using classical precursors like $\mathrm{Si}(\mathrm{OEt})_{4}$ (TEOS) in combination with organoalkoxysilanes like $\mathrm{RSi}(\mathrm{OEt})_{3}[1 \mathrm{~b}, 6]$.
The mechanism of the condensation reaction of silanols on a silica surface with hydrolysable $\mathrm{Si}-\mathrm{X}$ groups is still not fully understood. A very important factor is the number of silanol-groups available for the reaction [3]. Silica contains chemically and physically bound water $[1 \mathrm{~b}, 6 \mathrm{a}]$. Upon heating first dehydration (removal of physically bound water) and then dehydroxylation (condensation of neighbouring silanols and removal of water as the condensation product) take place $[1,6 c, 7]$.

Because of their reactivity and the stability of the resulting siloxane bonds, silanes containing hydrolysable alkoxy, halide, amide and carboxylate groups are commonly employed. Most reactive are $\mathrm{Si}-\mathrm{Hal}$ functions $(\mathrm{I}>\mathrm{Br}>\mathrm{Cl})$, with $\mathrm{Hal}=\mathrm{Cl}$ used almost exclusively. $\mathrm{Si}-\mathrm{O}_{2} \mathrm{CCH}_{3}$, SiOMe and SiOEt functions are less reactive [8]. Due to steric reasons only two $\mathrm{Si}$-X-groups of the $\mathrm{X}_{3} \mathrm{SiR}$ moieties react with the silica surface [1b]. The condensation of silanols on a completely dehydrated surface with $\mathrm{SiX}(\mathrm{X}=\mathrm{OR}, \mathrm{Hal})$ to form a siloxane only takes place when either small, but significant amounts of water or catalytic amounts of amines are present. If a slight excess of water is available on the silica surface, the $\mathrm{SiX}$-groups also react with each other. If the solution contains water, the silanes oligomerize and polymerize in solution before being grafted onto the silica surface [1b, 6a, 9-11]. 
Silanes containing alkoxy- and organoamino-groups are the most reactive silanes with respect to the reaction with surface silanols $[1 \mathrm{~b}, 12]$. The amino group also stabilizes alkoxy groups in the same molecule by forming internal hydrogen bonds, thereby preventing the condensation of the alkoxy groups in water-containing solution - a significant difference as compared to alkoxysilanes and chlorosilanes [1b, 13]. The most widely studied aminosilanes are 3-aminopropyltrimethoxysilane and 3-aminopropyltriethoxysilane [1b]. Numerous reports on modifications of silanol-containing surfaces of metal oxides with various silanes have been published, but to the best of our knowledge only one report relevant to the topic of this paper appeared, describing the use of 3-(2-aminoethylamino)propyltrimethoxysilan and 3-[2-(2-aminoethylamino)ethylamino]propyltrimethoxysilan for grafting on silica for column-liquid chromatography [12].

In this article we describe the drying procedure and the modification of different silica gels with various silanes, the characterization of the modified silicas employing scanning (SEM) and transmission (TEM) electron microscopy, energy-dispersive X-ray analysis (EDX), and elemental analysis. The preparation of heterogeneous cocatalysts, their testing in the polymerization of ethene using $\mathrm{Cp}_{2} \mathrm{ZrCl}_{2}$ and the characterization of the obtained polymers using SEM will be described in the following paper [14].

\section{Results and Discussion}

\section{Strategy}

The aim of our work was the preparation of tailormade carriers for the cocatalyst MAO (methylaluminoxane) [15]. For this application a porous carrier particle is needed, which can be fragmented during polymerization due to the growth of the polymer inside the particle [2a,b]. Carriers are therefore usually selected from porous silica of different particle and pore size with various, generally large surface areas ( $>100 \mathrm{~m}^{2} / \mathrm{g}$ ). The pore size of such silicas restricts the use of the total surface area to areas within pores which are wide enough to be accessible for the large MAOmolecules. MAO may then block the pores and so reduce the accessible surface area even further. Thus, in reality only small fractions of the total surface area are grafted by MAO.

To avoid these problems we have chosen spherical silica nano-particles Monospher $250^{\circledR}$ (Merck). These are dense silica spheres with an average diam- eter of $235 \mathrm{~nm}$ and a narrow particle size distribution of only 225 to $250 \mathrm{~nm}$ (determined by SEM), practically no pore volume, a surface area of $12 \mathrm{~m}^{2} / \mathrm{g}$ and $0.12 \mathrm{mmol} \mathrm{OH} / \mathrm{g}$. The whole surface area of these spheres is accessible to modification reactions, and problems like restricted pore size, diffusion limitations etc. are avoided. These particles can be filtered using a commercially available Teflon filter membrane with a pore size of $200 \mathrm{~nm}$, which allows the preparation of batches up to $200 \mathrm{~g}$. For comparison we used Snowtex 40 (Nissan), a spherical silica with diameters of 10-20 nm dispersed in water. Spray drying using commercial techniques resulted in mostly ringand sphere-shaped particles with sizes in the micrometer range. We also employed commercially available non-spherical Kieselgel 60 (Merck).

To generate a porous carrier particle starting from single, nano-particular silica spheres the surface modification has to meet two requirements: a) the spheres have to be interlinked to form a porous network, which is hard enough to survive handling and transport without too much erosion, but with interlinkages weak enough to be broken slowly during the polymerization by the growing polymer; b) various functional groups need to be introduced which upon modification with organometallic compounds will bind these components in different ways (physical or chemical grafting) to yield a non-leaching heterogeneous cocatalyst.

To fulfil these requirements we have chosen two types of organoalkoxysilanes as "spacers" and "linkers". As spacers we selected 3-aminopropyltrimethoxysilane (N1), 3-(2-aminoethylamino)propyltrimethoxysilane (N2) and 3-[2-(2-aminoethylamino) ethylamino]propyltrimethoxysilane (N3). Their amino groups react with the aluminium organyls to form Al$\mathrm{N}$ bonds, resulting in a chemisorption of the cocatalyst on the spacer-modified silica. After the pre-drying procedure for the silica gel $\left(150-160{ }^{\circ} \mathrm{C}, 8 \mathrm{~h}, 10^{-3}\right.$ $10^{-6}$ bar), small amounts of water remain on the silica surface. Upon reaction with the silica, the spacers are grafted onto the surface, with the residual water allowing the interlinking of the spacers on the surface of one sphere by a condensation of yet unreacted SiOMegroups and - depending on the ratio of spacer versus water and silanol - between spheres, resulting in interlinking of the spheres to give porous particles (Fig. 1).

As linkers we used 1,n-bis(alkoxysilyl)s, the bridging unit being a chain of different length containing different functional groups to vary the mode of interaction with the cocatalytically active aluminium organyls 

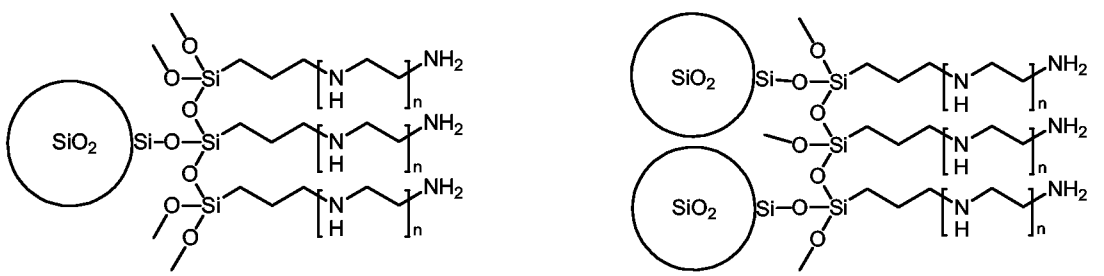

Fig. 1. Interlinking of aminosilanes on a silica sphere and between silica spheres (spacer N1: $n=0$, spacer $\mathrm{N} 2: \quad n=1$, spacer N3: $n=2$ ).
(Fig. 2). The linkers C8, PDMS and PPO bind the cocatalyst via physisorption, C3NC3 via chemisorption.

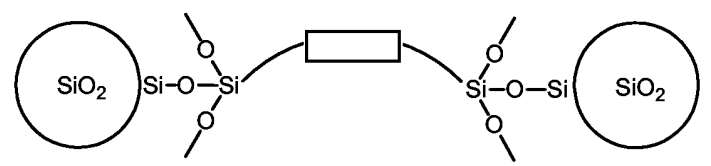

Fig. 2. Interlinking of silica spheres using linkers; mole of interaction of the central units $\square$ with aluminium organyls: $\square=-\left(\mathrm{CH}_{2}\right)_{8}-(\mathrm{C} 8$, van der Waals-interaction $)$, $-\left[\mathrm{Si}\left(\mathrm{CH}_{3}\right)_{2}-\mathrm{O}\right]_{\mathrm{n}}-(\mathrm{PDMS}$; weak donor-acceptor-interaction $\mathrm{O} \rightarrow \mathrm{Al}),-\left(\mathrm{CH}_{2}\right)_{3}-\left[\mathrm{O}-\mathrm{CH}_{2}-\mathrm{CH}\left(\mathrm{CH}_{3}\right)\right]_{5,5}-\mathrm{O}-\left(\mathrm{CH}_{2}\right)_{3}-\quad(\mathrm{PPO}$; strong donor-acceptor-interaction $\mathrm{O} \rightarrow \mathrm{Al}), \quad-\left(\mathrm{CH}_{2}\right)_{3}-\mathrm{NH}-$ $\left(\mathrm{CH}_{2}\right)_{3}-(\mathrm{C} 3 \mathrm{NC} 3$; Al-N bonding).

Upon reaction with the silica, one of the two silyl groups of the linkers reacts in the same way as the spacers. The second silyl-group then can react with the same or with another silica sphere, thereby interlinking the spheres to porous particles. The interlinking of spheres - although possible and observed also with the sole use of spacers - is more easily achieved using linkers due to the availability of a second hydrolysable alkoxysilyl group.

By using spacer and linker together and varying the combinations of spacer/linker and the molar ratios spacer:linker:silanol: water ( silanol = silanol groups on silica; water = total amount of water, vic. residual water on silica plus added water) we were able to vary the degree of surface modification (layer thickness, density of molecules, interlinking of the silica spheres) and the number and ratios of functional groups. In this way we could tune the surface and fragmentation properties of the prepared silica carriers, and after modification with cocatalysts we obtained highly active, nonleaching cocatalysts showing the desired fragmentation pattern during polymerization.

\section{Nomenclature of the prepared supports}

The nomenclature of the supports follows the scheme "silica(linker)spacer(preparation)". For the abbreviations of the silicas, linkers, spacers, and special procedures employed for the preparation see Table 1 .
Table 1. Nomenclature of the components for the supports.

\begin{tabular}{lll}
\hline Compound/method & Abbreviation \\
\hline Silica & Monospher 250 (Merck) & $\mathrm{M} 250$ \\
& Kieselgel 60 (Merck) & $\mathrm{K} 60$ \\
& Snowtex 40 (Nissan Chem.) & $\mathrm{S} 40$ \\
Linker & 1,8-Bis(triethoxysilyl)octane & $\mathrm{C} 8$ \\
& Bis[(3-trimethoxysilyl)propyl]amine & $\mathrm{C} 3 \mathrm{NC} 3$ \\
& Bis[(3-dimethoxymethylsilyl)propyl]- & \\
& polypropyleneoxide & $\mathrm{PPO}$ \\
& Methoxy-terminated polydimethylsiloxane & $\mathrm{PDMS}$ \\
Spacer & 3-Aminopropyl-trimethoxysilane & $\mathrm{N} 1$ \\
& 3-(2-Aminoethylamino)- & \\
& propyl-trimethoxysilane & $\mathrm{N} 2$ \\
& 3-[2-(2-Aminoethylamino)ethylamino]- & \\
& propyl-trimethoxysilane & $\mathrm{N} 3$ \\
Prepa- & without addition of water & $\mathrm{oW}$ \\
ration & with addition of water & $\mathrm{mW}$ \\
& low ratio/ high ratio silanes/silanol & $\mathrm{nB}, \mathrm{hB}$ \\
& reverse addition: 1. silanes, 2. silica & $\mathrm{R}$ \\
\hline
\end{tabular}

\section{Results}

The importance of the ratio silanol/linker/spacer/ water for the degree of surface modification has been demonstrated by preparing 29 different modified silica carriers.

The equivalent amount of water is especially important for the grafting of alkoxysilanes on silica. Surface modification was shown to take place in every preparation by performing SEM and TEM examinations. The degree of modification varied from barely visible on TEM-images to linkages of $20 \mathrm{~nm}$ width and carriers covered completely with excessive polysiloxane.

We have demonstrated that the degree of modification can be adjusted by varying one or all of the six variables given for a distinct silica: the amount of silanol (depending on the drying procedure of the silica), the equivalent amount of linker, the equivalent amount of spacer, the equivalent amount of water, the method of adding the water to the silica suspension, and the sequence of adding silica and silanes to the solvent. We altered every variable at least in one preparation, but focused our research on the amounts of water, linker and spacer using seven different silanes and three different silicas. As a result we were able to pre- 


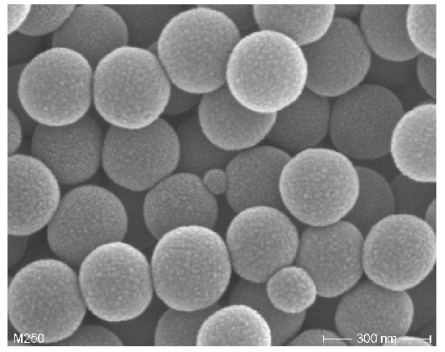

a)

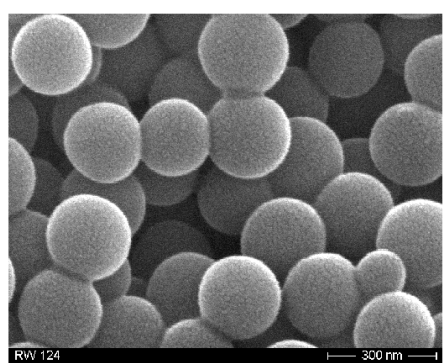

b)
Fig. 3. SEM images of a) M250N3 (mW), b) M250N3(oW) (100000fold magnification). pare modified silicas that can be used as carriers for very active heterogeneous cocatalysts for the polymerization of olefins.

\section{Reactivity of spacers and linkers in the condensation reaction}

The spacers N1, N2, N3 and the linker C3NC3 are aminosilanes which are able to form internal hydrogen bonds between the oxygen atoms of the alkoxy group and the hydrogen atoms of the amino groups thereby lending stability to solutions containing traces of water. They autocatalyse the condensation reaction between alkoxy and silanol groups, an important effect in the absence of water to achieve high grafting ratios. The reactivity slightly decreases in the order N3 $>\mathrm{N} 2>$ $\mathrm{N} 1>\mathrm{C} 3 \mathrm{NC} 3$. In the presence of water the stabilization comes into play, with the linker C3NC3 exhibiting the smallest $\mathrm{NH} /$ methoxy-ratio and therefore being the most reactive of the aminosilanes used. The linkers C8, PPO and PDMS show no autocatalysis in the condensation with silanols. In the absence of water they are therefore less reactive than $\mathrm{C} 3 \mathrm{NC} 3: \mathrm{N} 3>\mathrm{N} 2>$ $\mathrm{N} 1>\mathrm{C} 3 \mathrm{NC} 3>\mathrm{C} 8$, PPO, PDMS. In the presence of water oligo-/polymerization in solution occurs prior to the grafting of the silanes onto the silica, following the order $\mathrm{N} 3<\mathrm{N} 2<\mathrm{N} 1<\mathrm{C} 3 \mathrm{NC} 3<\mathrm{C} 8$, PPO, PDMS. As a result, the amount of spacers/linkers and the ratios of spacer : linker being actually grafted depend not only on the ratios employed in specific reactions, but are also dependent on the relative amount of water used in the modification reaction.

Consequently, addition of water to the silica suspension before adding the silanes was a crucial step in all the reactions. To achieve a uniform modification the water has to be as uniformly distributed over the particles as possible. To identify the best modification procedure we started with modifying M250 with N3 us- ing three variations: addition of water and silane to the suspended silica, addition of silane to the silica, and addition of silica to the silane solution.

\section{Spacer-modified silica}

For the preparation of $\mathrm{M} 250 \mathrm{~N} 3(\mathrm{~mW})$ equimolar amounts of water (silane: water $=1: 1$ ) were employed. The increase in mass in the reaction is very large $(26.3 \%)$. When preparing $\mathrm{M} 250 \mathrm{~N} 3(\mathrm{oW})$ with less water (silane: water $=1: 0.38$ ) a mass increase of only half of that of M250N3(mW) was observed. To change the order of addition, the silane was dissolved in the solvent and the silica added slowly to produce M250N3(oW,R). The mass increased to the same extent as with M250N3(mW), even though with M250N3(oW,R) 32\% more silane was employed. The slow addition of silica to the N3-solution appears to result in a much better surface coverage by the silane. This was unexpected, based on the following reasoning: When silica is suspended in toluene or heptane with intensive stirring and ultrasound irradiation, the agglomerates of the M250-spheres should leave only very few agglomerates of spheres intact. On the other hand, when silica is added to the N3-solution the agglomerates are not destroyed before reacting with N3, and therefore the reaction should take place on the smaller surface of the agglomerates as compared to the total surface of the separate spheres, resulting in a lower mass increase. However, this is not the case, so in some way $\mathrm{N} 3$ appears to act like a tenside, degrading the sphere agglomerates and covering the spheres to the maximum extent. Representative SEM images are depicted in Fig. 3.

The modifications of M250 with N1 (M250N1 $(\mathrm{mW})$ ) and with N2 (M250N2(mW)) show a correlation of the mass increase compared to M250N3(mW): With the methoxy-groups omitted, the molar masses 
are $172.33 \mathrm{~g} / \mathrm{mol}$ for $\mathrm{N} 3,86.19 \mathrm{~g} / \mathrm{mol}$ for $\mathrm{N} 1(50 \%$ of N3) and $129.26 \mathrm{~g} / \mathrm{mol}$ for $\mathrm{N} 2$ (75\% of N3), respectively. The mass increase for $\mathrm{M} 250 \mathrm{~N} 1(\mathrm{~mW})$ is $43 \%$ and that of $\mathrm{M} 250 \mathrm{~N} 2(\mathrm{~mW}) 70 \%$ of that of $\mathrm{M} 250 \mathrm{~N} 3(\mathrm{~mW})$, respectively. If the slight excess of water used for M250N1(mW) and M250N2(mW) is taken into account in combination with the difficulty of determining exactly the mass increase $(0.01$ to $0.3 \mathrm{~g}$ of the silica is usually lost during work-up), the correlation is quite satisfactory. A representative TEM image is depicted in Fig. 4.

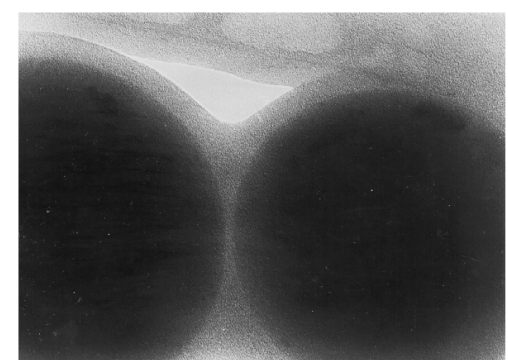

Fig. 4. TEM image of M250N2(mW) (400000-fold magnification).

The modification of K60 with N2 and N3 also correlates very well despite the porosity of the material. The mass increase upon preparation of $\mathrm{K} 60 \mathrm{~N} 2(\mathrm{~mW})$ is $63 \%$ of that of $\mathrm{K} 60 \mathrm{~N} 3(\mathrm{~mW}), 12 \%$ less than expected. However, if the slight excess of 0.15 equivalents of water used with $\mathrm{N} 3$ is considered, then the mass increase for $\mathrm{K} 60 \mathrm{~N} 2(\mathrm{~mW})$ is $74 \%$ of that of $\mathrm{K} 60 \mathrm{~N} 3(\mathrm{~mW})$, corresponding to the differences in molar mass of $\mathrm{N} 2$ and $\mathrm{N} 3$, N2 being $75 \%$ of that of N3 (molar masses without methoxy-groups). K60 thus reacts with $\mathrm{N} 2$ and $\mathrm{N} 3$ to the same extent if the same amount of water is present, and the effect of the porosity of K60 is negligible.

\section{Linker-modified silica}

The internal chain lengths of the linkers $\mathrm{C} 3 \mathrm{NC} 3$ and C8 are short with 7 and 8 atoms, respectively. Linkers with longer alkyl and aminoalkyl chains are commercially not available. The chain length of the linkers PPO and PDMS has been calculated from their molar masses. With a molar mass of $600 \mathrm{~g} / \mathrm{mol}$ and the known structure of PPO its chain consists of 5.5 repeating units and contains 6.5 oxygen atoms, which may function as donors. PDMS with a molar mass of $17000 \mathrm{~g} / \mathrm{mol}$ has a chain with 227 repeating units and 228 oxygen atoms, which are poor donors as compared to PPO due to special interaction with the neighbouring silicon atoms.

For the modification of S40 with PDMS, $\mathrm{Et}_{2} \mathrm{NH}$ was added as a catalyst. Subsequently, HDMS was introduced to endcap the unreacted silanol groups on silica. With all linkers two carriers (modified silica) were prepared using a low and a high silanol:linker:water ratio of $1: 1: 1.55$ and $1: 15: 15$, respectively. In the case of PPO the high ratio was achieved by saturating the suspension purging it with a stream of humid nitrogen. When using PDMS with its high molar mass the amount of linker was reduced drastically.

Very surprisingly each of the modifications - except the one using S40 - resulted in a net loss of weight, although a surface modification clearly had taken place as demonstrated by the SEM and TEM images, which showed interlinking of the silica spheres via polysiloxane.

With $\mathrm{M} 250(\mathrm{C} 8)(\mathrm{oW})$ the modification/interlinking was not obvious from SEM results, but TEM showed interlinkages of 25-40 nm width. For M250(C8)(mW) there is a clear evidence of surface modification resulting in chemical bonds between the spheres: Fig. 5

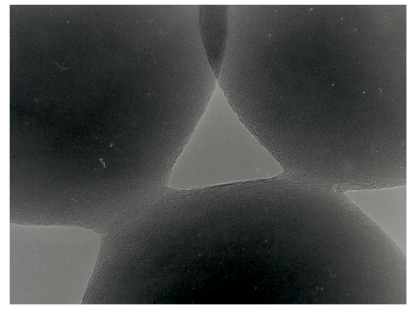

a)

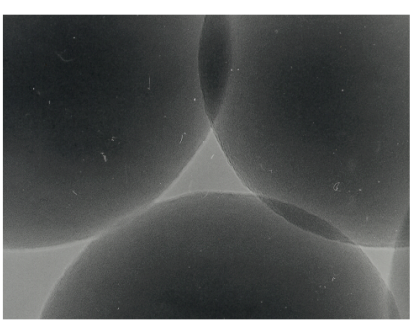

b)

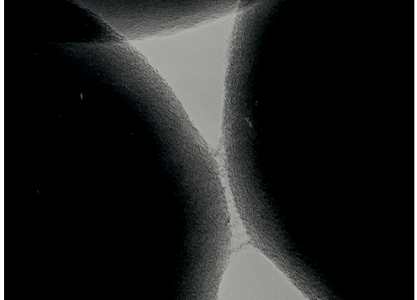

c)

Fig. 5. TEM images of a) M250(C8)(oW) (620000-fold magnification), b) M250(C8)(mW) (400000-fold magnification) before the energy input of the electron beam and c) M250(C8)(mW) (400000-fold magnification) after the energy input of the electron beam. 
shows the stretching of the interlinkages upon electron bombardement without a breaking of the contacts.

M250(C3NC3)(oW) was less extensively modified than M250(C8)(oW), but M250(C3NC3)(mW) showed clear interlinking (Fig. 6). M250(PDMS)(nB) and M250(PDMS)(oW) as well as M250(PPO)(oW) and $\mathrm{M} 250(\mathrm{PPO})(\mathrm{mW})$ are not very different, with interlinkages of $35-55 \mathrm{~nm}$.

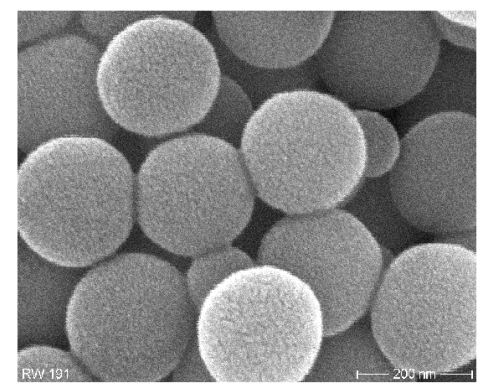

Fig. 6. SEM image of M250(C3NC3)(mW) (150000-fold magnification).

\section{Silica modified with combinations of linker and spacer}

With M250 combinations of N1 and N3 with all linkers and of $\mathrm{N} 3$ with $\mathrm{C} 3 \mathrm{NC} 3$ were prepared.

Combinations of $\mathrm{C} 8$ with N1. This combination is the most unfavourable in terms of the reactivity difference of the silanes towards the condensation reaction with surface silanol groups. Due to the small amount of $\mathrm{C} 8$ used in the preparation of $\mathrm{M} 250(\mathrm{C} 8) \mathrm{N} 1(\mathrm{oW})$, the large excess of $\mathrm{N} 1$ relative to $\mathrm{C} 8(\mathrm{OH}: \mathrm{C} 8: \mathrm{N} 1:$ water $=1: 0.4: 5.4: 1.55)$ and the much higher reactivity of $\mathrm{C} 8$ compared to N1, probably all of the $\mathrm{C} 8$ has been grafted. For the preparation of $\mathrm{M} 250(\mathrm{C} 8) \mathrm{N} 1(\mathrm{hB})$ larger amounts of silanes and a small amount of water were employed $(\mathrm{OH}: \mathrm{C} 8: \mathrm{N} 1:$ water $=1: 4: 20: 1.55)$. $\mathrm{C} 8$ should thus be grafted predominantly, N1 only to a smaller extent.

Combinations of PPO with N1. Three combinations of the linker PPO with spacer N1 were prepared. The combination of low PPO content with a ten-fold excess of $\mathrm{N} 1$ and with only a slight excess of water compared to silanol (M250(PPO)N1(oW); $\mathrm{OH}: \mathrm{PPO}: \mathrm{N} 1:$ water $=1: 1.03: 10.3: 1.55)$ resulted in a modified silica with a $\mathrm{C} / \mathrm{N}$-ratio of 6.33 . Although there was no mass increase, surface modification took place as can be seen from the SEM images. M250(PPO)N1(hB) prepared using a larger excess of PPO and N1 but only twice the amount of water compared to PPO, gave a C/N-ratio of 7.53. The surface modification is less marked than with M250(PPO)N1(oW). For M250(PPO)N1(mW) the standard ratio of $\mathrm{OH}: \mathrm{PPO}: \mathrm{N} 1:$ water $=1: 5: 15: 20$ was employed. The carrier produced had a $\mathrm{C} / \mathrm{N}$-ratio of 3.21, smaller than the other two samples. The surface modification is shown in Fig. 7.

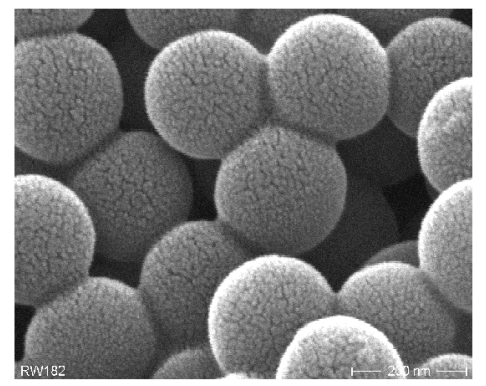

Fig. 7. SEM image of M250(PPO)N1(mW) (150000-fold magnification ).

Combinations of PDMS with N1. With PDMS only one combination with a spacer was prepared. The M250(PDMS)N1(nB) product with a C/N-ratio of 9.23 showed a surface modification with interlinkages of $50-60 \mathrm{~nm}$ width (Fig. 8).

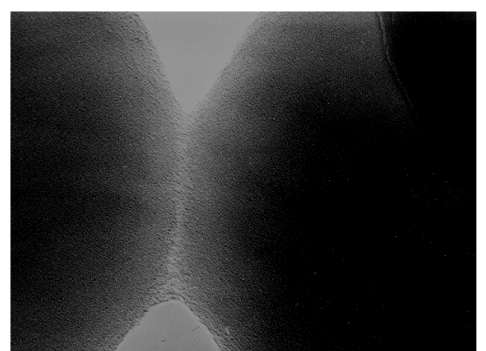

Fig. 8. TEM image of M250(PDMS)N1(nB) (620000-fold magnification).

Combinations of C3NC3 with N1. The combination of linker C3NC3 with spacer N1 is the combination most suited to evaluate the properties of a heterogeneous cocatalyst depending on the linker: spacer ratio and the optimum amount of water, because $\mathrm{C} 3 \mathrm{NC} 3$ and the spacers, especially N1, are most similar regarding structure and reactivity towards the condensation reaction. Thus the ratios were altered extensively starting from low to high linker/spacer/water contents. Four variations with a ratio of $\mathrm{OH}: \mathrm{C} 3 \mathrm{NC} 3:$ spacer $=$ $1: 5: 15$ were prepared with water equivalents of 15 , 20, 25.8, and large excess. After each reaction an increase in mass was observed for the products, but 


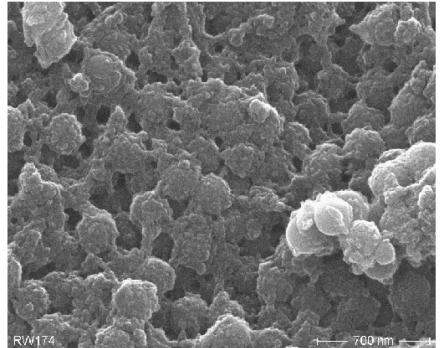

a)

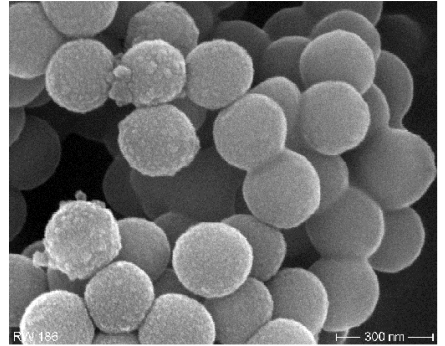

b)

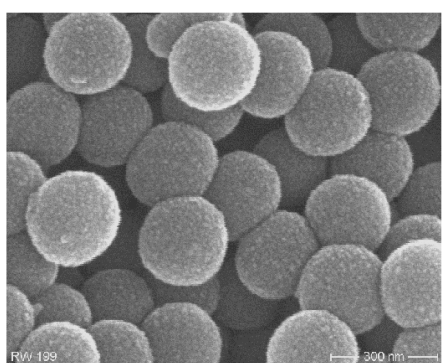

c)

Fig. 9. TEM-images of a) M250(C3NC3)N1(mW)A (45000-fold magnification), b) M250(C3NC3)N1(mW)B (90000-fold magnification) and c) M250(C3NC3)N1(mW)C (100000-fold magnification).

with the same $\mathrm{OH}: \mathrm{C} 3 \mathrm{NC} 3$ : spacer ratios the mass increase was only half when reducing the equivalents of water from $25.8(\mathrm{M} 250(\mathrm{C} 3 \mathrm{NC} 3) \mathrm{N} 1(\mathrm{~mW}) \mathrm{A})$ to $20(\mathrm{M} 250(\mathrm{C} 3 \mathrm{NC} 3) \mathrm{N} 1(\mathrm{~mW}) \mathrm{B})$ (A and $\mathrm{B}$ and $\mathrm{C}$ (below) are nomenclature marks for the sake of distinction). With a further reduction of the equivalents of water to $15(\mathrm{M} 250(\mathrm{C} 3 \mathrm{NC} 3) \mathrm{N} 1(\mathrm{~mW}) \mathrm{C})$, the mass increase was almost the same as observed for M250(C3NC3)N1(mW)A. The SEM image of M250(C3NC3)N1(mW)C (Fig. 9c) shows very uniform surface modification with only very little excessive condensed polysiloxane (in only $10 \%$ of the samples some polysiloxane can be detected), whereas M250(C3NC3)N1(mW)A (Fig. 9a) shows M250-agglomerates completely covered with irregularly condensed polysiloxane with barely visible M250-spheres. Thus, the high degree of surface modification in $\mathrm{M} 250(\mathrm{C} 3 \mathrm{NC} 3) \mathrm{N} 1(\mathrm{~mW}) \mathrm{C}$ is the result of thick but uniform surface layers retaining the spherical shape of the individual M250spheres. The degree of interlinking is comparable to M250(C3NC3)N1(mW)B (Fig. 9b), which is a carrier with little condensed polysiloxane (in approx. $20 \%$ of the sample) on the interlinked M250-spheres. When modified with MAO (oligomeric methylaluminoxane) $\mathrm{M} 250(\mathrm{C} 3 \mathrm{NC} 3) \mathrm{N} 1(\mathrm{~mW}) \mathrm{C}$ showed the highest activity in the polymerization of ethylene using $\mathrm{Cp}_{2} \mathrm{ZrCl}_{2}$ of all heterogeneous cocatalysts prepared; see Part II, describing the modification of these carriers with various aluminium alkyl compounds and their testing in the polymerization of ethylene using zirconocene dichloride [14].

One combination of $\mathrm{C} 3 \mathrm{NC} 3$ with $\mathrm{N} 3$ was prepared, M250(C3NC3)N3(mW), trying to maximize the amount of water without having a separate layer of water in the reaction mixture. To achieve this, ni- trogen was purged through distilled water and then through the suspension of M250 in toluene for six hours. Partially evaporated solvent was then replaced and the modification reaction was carried out as usual. The mass increased only by $25.4 \%$ (less than with M250(C3NC3)N1(mW)A), but the SEM and TEM images showed M250-spheres with a thick, but very even surface layer of grafted polysiloxane $(10-30 \mathrm{~nm}$ layer thickness). Saturation with water is therefore to be performed preferably by using a continuous stream of humid gas instead of by simply adding water using e.g. a syringe (M250(C3NC3)N1(mW)A). The surface morphology has a great impact on the polymerization activity of the corresponding heterogeneous cocatalysts.

S40 was modified using $\mathrm{C} 3 \mathrm{NC} 3 / \mathrm{N} 1$ to yield S40(C3NC3)N1(mW). As described for S40(PDMS) (oW), HMDS was added to encap unreacted silanols. The mass increased by $10.5 \%$ but the TEM images showed the same micrometer-scale particles as with S40(PDMS)(oW).

\section{Conclusions}

Surface-modified silica has been prepared by reacting silica particles with various alkoxysilanes as spacers and linkers. The crucial issues regarding the modification were the ratio silanol:spacer:linker: water and the relative reactivity of the alkoxysilanes in the condensation with silanol groups on silica. The coverage with functional groups and bridging units $\left[(\mathrm{NH})_{n}\right.$, $\left.-\left(\mathrm{SiMe}_{2} \mathrm{O}\right)_{227^{-}},-\left(\mathrm{CH}_{2} \mathrm{C}\left(\mathrm{CH}_{3}\right) \mathrm{O}\right)_{7^{-}},-\left(\mathrm{CH}_{2}\right)_{8}-\right]$ can be varied in a wide range. Depending on the relative amounts of silanes and the preparative procedure (silica added to a solution of silanes, or silanes added to suspended silica; concentration of silica and/or silanes), the thickness and the quality of the layer 
grafted on the surface of the silica particles can be tuned. The surface morphologies determine the fragmentation pattern of the particles and the polymerization activity of the heterogeneous cocatalysts with MAO grafted on these modified silicas (described in Part II) [14].

Other possible uses of the new modified silicas are in liquid chromatography and ion exchange chromatography, or as filtering aids. For these potential applications the possible variations in hydrophilicity/hydrophobicity of the silicas ranging from almost water-soluble (M250(C3NC3)N3(mW)) to water-insoluble $(\mathrm{M} 250(\mathrm{C} 8)(\mathrm{mW}))$ are of particular interest. The products can also be used as additives to reinforce plastics since the surface modification makes the particles compatible with plastics.

\section{Experimental Section}

All reactions and work-up procedures including filtration and centrifugation were performed using Schlenk techniques. For filtration a commercially available glass filter with Teflon filter membranes (200 nm pore size) was remodelled for use under inert atmosphere to filter suspensions of Monospher 250. Suspensions of Snowtex 40 were centrifuged at $2000 \mathrm{rpm} / 45 \mathrm{~min}$ in Schlenk vessels in a Hettich centrifuge.

The solvents $n$-pentane, $n$-hexane, $n$-heptane and toluene were dried under inert atmosphere using sodium metal with benzophenone as indicator. The solvents were refluxed until the colour changed to purple and were then distilled and used freshly. Diethylamine was distilled, stirred with sodium metal, distilled again and then stored under nitrogen over molecular sieve $4 \AA$. The silanes 3-aminopropyltrimethoxysilane (N1), 3-(2-aminoethylamino)propyltrimethoxysilane (N2), 3-[2-(2-aminoethylamino)ethylamino]propyltrimethoxysilane (N3), 1,8-bis(triethoxysilyl)octane (C8), bis[(3-trimethoxysilyl)propyl]amine (C3NC3), bis[3(dimethoxymethylsilyl)propyl]polypropylene oxide (PPO) and methoxy-terminated polydimethylsiloxane (PDMS) were used as received (contents 95 to $>99 \%$ varying with batches; $90 \%$ for N3).

The glass apparatus were evacuated, heated to above $100{ }^{\circ} \mathrm{C}$, allowed to cool and then flushed with pure nitrogen.

Kieselgel 60 (commercial product) and Monospher 250 were donated from Merck, Darmstadt, Snowtex 40 from Nissan Chemical Ind., Japan. The properties of the silica gels and drying conditions employed are listed in Tables 2 and 3. Active Si-OH groups at the surface of M250 have been calculated to $6-7 \mathrm{OH} / \mathrm{nm}^{2}$ and $0.12 \mathrm{mmol} \mathrm{OH} / \mathrm{g}$. By using this in combination with a calculated surface area of $300 \mathrm{~m}^{2}$ at $10 \mathrm{~nm}$ diameter the $\mathrm{Si}-\mathrm{OH}$ group content of $\mathrm{S} 40$ was
Table 2. Properties of the employed silica.

\begin{tabular}{llcccc}
\hline Silica & $\begin{array}{l}\text { Average } \\
\text { particle } \\
\text { size }[\mathrm{nm}]\end{array}$ & $\begin{array}{c}\text { Particle size } \\
\text { distribution } \\
{[\mathrm{nm}]}\end{array}$ & $\begin{array}{c}\text { Specific } \\
\text { surface } \\
{\left[\mathrm{m}^{2} / \mathrm{g}\right]}\end{array}$ & $\begin{array}{c}\text { Pore } \\
\text { volume } \\
{[\mathrm{ml} / \mathrm{g}]}\end{array}$ & $\begin{array}{c}\text { Pore } \\
\text { diameter } \\
{[\mathrm{nm}]}\end{array}$ \\
\hline M250 & 235 & $225-245$ & 12 & 0 & 0 \\
S40 & not known & $10-20$ & $\sim 300$ & 0 & 0 \\
K60 & not known & $63-200$ & $\sim 490$ & $0.71-0.78$ & 6 \\
\hline
\end{tabular}

Table 3. Drying conditions for the employed silica.

\begin{tabular}{llll}
\hline Silica & $\begin{array}{c}\text { Lot } \\
\text { No. }\end{array}$ & Drying conditions & $\begin{array}{l}\text { Water content } \\
\text { [wt. \%] }\end{array}$ \\
\hline M250 & 1 & $150^{\circ} \mathrm{C} / 6 \mathrm{~h} /<0.01 \mathrm{mbar}$ & 0.8 \\
$\mathrm{M} 250$ & 2 & $150^{\circ} \mathrm{C} / 8 \mathrm{~h} /<0.01 \mathrm{mbar}$ & 0.335 \\
$\mathrm{M} 250$ & 3 & $150^{\circ} \mathrm{C} / 6 \mathrm{~h} /<0.01 \mathrm{mbar}$ & 1.6 \\
$\mathrm{M} 250$ & 4 & $165^{\circ} \mathrm{C} / 6 \mathrm{~h} /<0.01 \mathrm{mbar}$ & 0.87 \\
$\mathrm{~S} 40$ & 1 & $130^{\circ} \mathrm{C} / 1 \mathrm{~h} /<0.01 \mathrm{mbar}$ and & 0.8 \\
& & $155^{\circ} \mathrm{C} / 7 \mathrm{~h} /<0.01 \mathrm{mbar}$ & \\
$\mathrm{K} 60$ & 1 & $155^{\circ} \mathrm{C} / 5 \mathrm{~h} /<0.01 \mathrm{mbar}$ & 5.6 \\
\hline
\end{tabular}

$2.99 \mathrm{mmol} \mathrm{OH} / \mathrm{g}$. (Using the value of $4.6 \mathrm{OH} / \mathrm{nm}^{2}$, the numbers are $0.092 \mathrm{mmol} \mathrm{OH} / \mathrm{g}$ for $\mathrm{M} 250$ and $2.3 \mathrm{mmol} \mathrm{OH} / \mathrm{g}$ for $\mathrm{S} 40$ ). The numbers 0.12 and $2.99 \mathrm{mmol} \mathrm{OH} / \mathrm{g}$ were used for the calculation of the molar ratios.

Elemental analysis was performed using a Perkin-Elmer CHNS/O-analyser 2400, series II; for SEM and EDX a Hitachi S-4000 field emission scanning electron microscope (FESEM) was used with 7-20 keV energies, with the samples sputtered with a 2-4 nm thick gold or Pt/Pd-layer; for TEM a Phillips CM200 transmission electron microscope with $200 \mathrm{keV}$ was employed; the samples were deposited on the carbon hole foil by evaporating a hexane suspension; the pictures were taken photographically and then enlarged by a factor of two.

General preparative procedure. 5 to $50 \mathrm{~g}$ of silica was suspended in 50 to $150 \mathrm{ml}$ of toluene or heptane with intensive stirring and applying ultrasound for 20 minutes. For preparations with added water the calculated amount of water (distilled twice) was added over a period of 5 to $20 \mathrm{~min}$ using a syringe with a very thin needle. After 30 min of stirring the silanes were added via syringe or dropping funnel as mixture or as solution in a small amount of solvent. The suspension was heated to reflux for 8 to $64 \mathrm{~h}$; the azeotrope methanol/solvent or ethanol/solvent was distilled off and the remaining suspension filtered using a Teflon membrane (for M250), or a D4 glass frit (for K60), or centrifuged (for S40), and washed with solvent in 3 to 5 portions of in total up to $120 \mathrm{ml}$ heptane, hexane or pentane (hexane preferred, for the last washing pentane). The residue was then dried in vacuo until pressures of less than 0.03 mbar were reached. The modified silicas are colourless powders, slightly hygroscopic and - depending on the silanes used for the modification hydrophilic or hydrophobic. Some are highly electrostatic.

Tables 4 and 5 list all prepared modified silicas with their nomenclature, the employed equivalent ratios of 
Table 4. Modified silicas: M250 and K60 - equivalent ratios and characterization.

\begin{tabular}{|c|c|c|c|c|c|c|c|c|}
\hline $\begin{array}{l}\text { Modified } \\
\text { silica }\end{array}$ & Linker & Spacer & $\begin{array}{l}\text { Equivalents } \\
\text { silanol : linker : } \\
\text { spacer : water }\end{array}$ & $\begin{array}{l}\text { Employed } \\
\text { silica } \\
{[\mathrm{g}]}\end{array}$ & $\begin{array}{c}\text { Increase of mass } \\
\text { after reaction } \\
{[\mathrm{g}]}\end{array}$ & $\begin{array}{c}\text { Increase } \\
\text { of mass } \\
{[\%]}\end{array}$ & $\begin{array}{l}\mathrm{C} / \mathrm{H} / \mathrm{N} \text {-content } \\
{[\mathrm{w} \%]}\end{array}$ & $\begin{array}{l}\text { Characterization } \\
\text { methods used }\end{array}$ \\
\hline$\overline{\mathrm{M} 250 \mathrm{~N} 1(\mathrm{~mW})}$ & - & N1 & $1: 0: 10.4: 10.5$ & 41.12 & 4.65 & 11.3 & $6.52 / 1.87 / 2.74$ & SEM \\
\hline $\mathrm{M} 250 \mathrm{~N} 2(\mathrm{~mW})$ & - & $\mathrm{N} 2$ & $1: 0: 10: 12.6$ & 56.12 & 10.30 & 18.4 & $9.05 / 2.44 / 3.72$ & TEM \\
\hline M250N3(oW) & - & N3 & $1: 0: 10: 3.8$ & 26.06 & 3.43 & 13.2 & $8.46 / 2.17 / 2.90$ & SEM \\
\hline M250N3(oW,R) & - & N3 & $1: 0: 13.2: 3.7$ & 37.96 & 10.27 & 27.0 & $12.78 / 2.77 / 4.70$ & SEM \\
\hline M250N3(mW) & - & N3 & $1: 0: 10: 10$ & 59.02 & 16.5 & 26.3 & $15.53 / 3.41 / 3.50$ & SEM \\
\hline $\mathrm{M} 250(\mathrm{C} 8)(\mathrm{oW})$ & $\mathrm{C} 8$ & - & $1: 1: 0: 1.55$ & 36.63 & - & - & $2.45 / 0.79 / 0.35$ & SEM, TEM \\
\hline $\mathrm{M} 250(\mathrm{C} 8)(\mathrm{mW})$ & $\mathrm{C} 8$ & - & $1: 15: 0: 15$ & 30.43 & - & - & $2.47 / 1.11 / 0.39$ & SEM, TEM \\
\hline M250(C3NC3)(oW) & C3NC3 & - & $1: 1: 0: 1.55$ & 34.93 & - & - & $2.90 / 0.97 / 0.41$ & SEM, TEM \\
\hline $\mathrm{M} 250(\mathrm{C} 3 \mathrm{NC} 3)(\mathrm{mW})$ & $\mathrm{C} 3 \mathrm{NC} 3$ & - & $1: 15: 0: 15$ & 43.18 & - & - & $5.85 / 1.76 / 0.45$ & SEM \\
\hline M250(PPO)(oW) & PPO & - & $1: 1.03: 0: 1.55$ & 31.00 & - & - & $3.00 / 0.96 / 0.29$ & SEM \\
\hline M250(PPO)(mW) & PPO & - & $1: 15: 0:$ saturated & 41.60 & - & - & $3.46 / 1.13 / 0.17$ & SEM \\
\hline M250(PDMS)(nB) & PDMS & - & $1: 0.186: 0: 1.55$ & 25.81 & - & - & $2.42 / 0.99 / 0.25$ & SEM, TEM \\
\hline M250(PDMS)(oW) & PDMS & - & $1: 1: 0: 7.4$ & 24.37 & - & - & $3.23 / 1.24 / 0.24$ & SEM \\
\hline M250(C8)N1(oW) & $\mathrm{C} 8$ & N1 & $1: 0.4: 5.4: 1.55$ & 36.34 & 0.94 & 2.6 & $4.25 / 1.14 / 0.67$ & SEM \\
\hline $\mathrm{M} 250(\mathrm{C} 8) \mathrm{N} 1(\mathrm{hB})$ & $\mathrm{C} 8$ & N1 & $1: 4: 20: 1.55$ & 26.97 & - & - & $3.14 / 0.98 / 0.47$ & SEM \\
\hline M250(C3NC3)N1(oW) & $\mathrm{C} 3 \mathrm{NC} 3$ & N1 & $1: 0.4: 5.4: 1.55$ & 33.28 & 0.82 & 2.5 & $4.81 / 1.22 / 0.92$ & SEM \\
\hline M250(C3NC3)N1(hB) & $\mathrm{C} 3 \mathrm{NC} 3$ & N1 & $1: 4: 22: 7.4$ & 45.88 & 2.54 & 5.5 & $6.50 / 1.63 / 1.08$ & SEM \\
\hline $\mathrm{M} 250(\mathrm{C} 3 \mathrm{NC} 3) \mathrm{N} 1(\mathrm{~mW}) \mathrm{A}$ & $\mathrm{C} 3 \mathrm{NC} 3$ & N1 & $1: 5: 15: 25.8$ & 32.26 & 10.48 & 32.5 & $11.99 / 2.59 / 2.78$ & SEM \\
\hline $\mathrm{M} 250(\mathrm{C} 3 \mathrm{NC} 3) \mathrm{N} 1(\mathrm{~mW}) \mathrm{B}$ & C3NC3 & N1 & $1: 5: 15: 20$ & 31.85 & 5.31 & 16.7 & $10.47 / 3.02 / 2.12$ & SEM \\
\hline $\mathrm{M} 250(\mathrm{C} 3 \mathrm{NC} 3) \mathrm{N} 1(\mathrm{~mW}) \mathrm{C}$ & C3NC3 & N1 & $1: 5: 15: 15$ & 24.55 & 7.09 & 28.9 & $10.13 / 2.59 / 2.81$ & SEM \\
\hline M250(C3NC3)N3(mW) & $\mathrm{C} 3 \mathrm{NC} 3$ & $\mathrm{~N} 3$ & $1: 5: 15:$ saturated & 47.36 & 12.04 & 25.4 & $11.74 / 2.87 / 3.43$ & SEM, TEM \\
\hline M250(PPO)N1(oW) & PPO & N1 & $1: 1.03: 10.3: 1.55$ & 31.11 & - & - & $6.01 / 1.59 / 0.95$ & SEM \\
\hline M250(PPO)N1(hB) & PPO & $\mathrm{N} 1$ & $1: 3.7: 20: 7.4$ & 20.99 & - & - & $4.52 / 1.31 / 0.60$ & SEM \\
\hline M250(PPO)N1(mW) & PPO & N1 & $1: 5: 15: 20$ & 32.67 & 5.93 & 18.2 & $7.83 / 2.01 / 2.44$ & SEM \\
\hline M250(PDMS)N1(nB) & PDMS & N1 & $1: 0.186: 3.5: 3.7$ & 47.84 & - & - & $4.34 / 1.20 / 0.47$ & SEM, TEM \\
\hline $\mathrm{K} 60 \mathrm{~N} 2(\mathrm{~mW})$ & - & $\mathrm{N} 2$ & $?: 0: 1.54: 1$ & 28.53 & 9.79 & 34.3 & $11.48 / 2.19 / 5.20$ & SEM \\
\hline $\mathrm{K} 60 \mathrm{~N} 3(\mathrm{~mW})$ & - & N3 & $?: 0: 1: 1.15$ & 27.83 & 15.14 & 54.4 & $15.23 / 2.52 / 8.29$ & SEM \\
\hline
\end{tabular}

Table 5. Modified silicas: S40 - equivalent ratios and characterization.

\begin{tabular}{|c|c|c|c|c|c|c|c|c|}
\hline $\begin{array}{l}\text { Modified } \\
\text { silica }\end{array}$ & Linker & Spacer & Equivalents & $\begin{array}{l}\text { Employed } \\
\text { silica } \\
{[\mathrm{g}]}\end{array}$ & $\begin{array}{c}\text { Increase of mass } \\
\text { after reaction } \\
{[\mathrm{g}]}\end{array}$ & $\begin{array}{c}\text { Increase } \\
\text { of mass } \\
{[\%]}\end{array}$ & $\begin{array}{l}\mathrm{C} / \mathrm{H} / \mathrm{N} \text {-content } \\
{[\mathrm{w} \%]}\end{array}$ & $\begin{array}{l}\text { Characterization } \\
\text { methods used }\end{array}$ \\
\hline$\overline{\mathrm{S} 40(\mathrm{PDMS})(\mathrm{oW})}$ & PDMS & - & $\begin{array}{l}\text { silanol : PDMS : } \\
\text { water : HDMS } \\
1: 0.1: 0.15: 1.05\end{array}$ & 4.89 & 0.29 & 5.9 & $11.11 / 3.38 / 0.50$ & TEM \\
\hline $\mathrm{S} 40(\mathrm{C} 3 \mathrm{NC} 3) \mathrm{N} 1(\mathrm{~mW})$ & $\mathrm{C} 3 \mathrm{NC} 3$ & N1 & $\begin{array}{l}\text { silanol : } \mathrm{C} 3 \mathrm{NC} 3: \\
\mathrm{N} 1: \text { water }: \mathrm{HMDS} \\
1: 1: 0.1: 1: 1.05\end{array}$ & 4.30 & 0.45 & 10.5 & $4.57 / 1.10 / 0.93$ & TEM \\
\hline
\end{tabular}

silanol: linker:spacer: water, the mass increase ("yield") and the characterization methods used. The types and amounts of starting material and solvent and the reactions time are listed in Tables 6 and 7. Both tables are to read as demonstrated for the preparation of M250N2(mW) (example 2 in Tab. 6: $56.12 \mathrm{~g}$ of M250 (lot \#1, $6.74 \mathrm{mmol}$ silanol) was suspended in $100 \mathrm{ml}$ of toluene and $50 \mathrm{ml}$ of heptane. $1.08 \mathrm{ml}(60 \mathrm{mmol})$ of bidistilled water was added and the suspension stirred for 30 minutes. Then $14.9 \mathrm{ml} \mathrm{N} 2$ spacer (98\% purity, $67.4 \mathrm{mmol}$ ) was added within 10 minutes and the mixture was stirred for $2 \mathrm{~h}$ at room temperature and for $12 \mathrm{~h}$ at reflux temperature. $40 \mathrm{ml}$ of azeotrope was distilled off. The suspension was filtered and the remaining solid was washed three times with toluene and four times with pentane.
The mass of the solid increased by $18.4 \mathrm{wt} . \%$ yielding $10.3 \mathrm{~g}$ of M250N2(mW).

\section{Acknowledgements}

This work was supported by the Fonds der Chemischen Industrie, the Bundesministerium für Bildung und Forschung (BMBF 03C0295A+B), the Deutsche Forschungsgemeinschaft (Graduiertenkolleg "Synthetische, mechanistische und reaktionstechnische Aspekte von Metallkatalysatoren”) and the Merck KgaA. We thank Dipl.-Ing. U. Gernert (TU Berlin) for the SEM-examinations and Dr. M.-M. Pohl (Institut für Angewandte Chemie Adlershof (ACA), Berlin) for the TEMexaminations, as well as Dr. T. Eberle and Dr. K. Köhler 

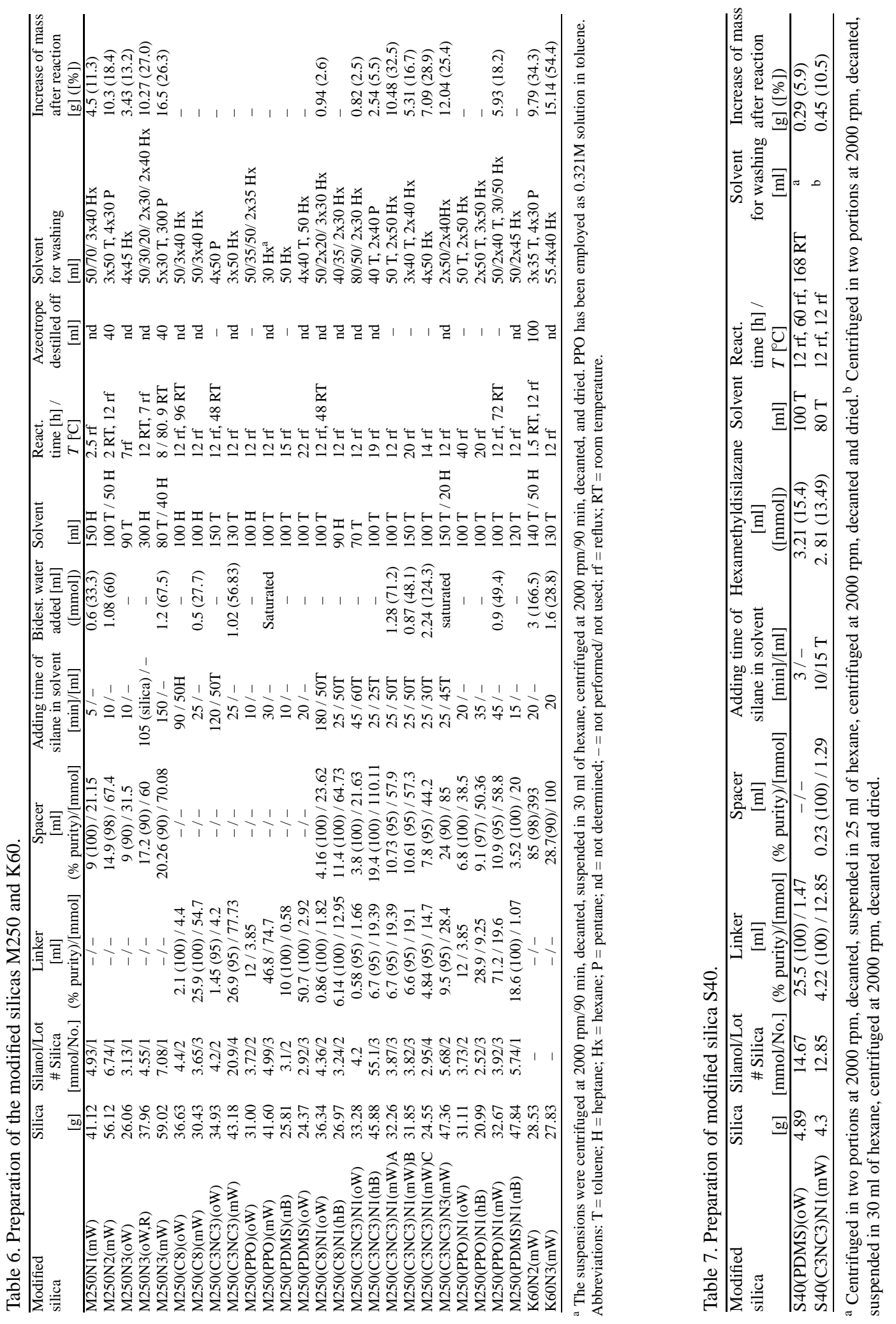
(Merck, Darmstadt), for helpful discussions during our joint research project. Monospher 250 and Kieselgel 60 were kindly donated by Merck, Darmstadt, and Snowtex 40 by Nissan Chemical Ind., Japan.
[1] a) G. Ertl, H. Knözinger, J. Weitkamp, Handbook of Heterogeneous Catalysis, Vol. 1, Ch. 2, Wiley-VCH, Weinheim (1997); b) E. F. Vansant, P. van der Voort, K. C. Vrancken, Characterization and Chemical Modification of the Silica Surface; Studies in Surface Science and Catalysis, Vol. 93, Elsevier, Amsterdam (1995).

[2] a) W. Kaminsky, Metalorganic Catalysts for Synthesis and Polymerization, Ch. 1, Springer, Berlin (1999); b) R. Blom, A. Follestad, E. Rytter, M. Tilset, M. Ystenes, Organometallic Catalysts and Olefin Polymerization; Catalysts for a New Millenium, Springer, Berlin (2001); c) J. F. Le Page, Applied Heterogeneous Catalysis - Design, Manufacture, Use of Solid Catalysts, Editions Technip, Paris (1987); d) E. Gallei, E. Schwab, Catal. Today 51, 535 (1999); e) Y. Iwasawa, in Y. Iwasawa (ed.): Tailored Metal Catalysts, Reidel, Dordrecht (1986).

[3] F. D. Osterholz, E. R. Pohl, in K.L. Mittaled (ed.): Silanes and Other Coupling Agents, 119, VSP, Utrecht (1992).

[4] E. I. Ko, in G. Ertl, H. Knözinger, J. Weitkamp (eds): Handbook of Heterogeneous Catalysis, Vol. 1, Ch. 2.1.4, Wiley-VCH, Weinheim (1997).

[5] a) J. J. Pesek, I.E. Leigh, Chemically Modified Surfaces, Royal Soc. Chem., Cambridge (1994); b) H. A. Mottola, J.R. Steinmetz, Chemically Modified Surfaces, Royal Soc. Chem., Cambridge (1992).

[6] a) C. J. Brinker, G. W. Scherer, Sol-Gel Science, Academic Press, New York (1989); b) W. Stöber, A. Fink, E. Bohn, J. Colloid Interface Sci. 26, 62 (1968); c) W. K. Thompson, Proc. Brit. Ceramic Soc. 5, 143 (1965).

[7] G. Ertl, H. Knözinger, J. Weitkamp, Handbook of Heterogeneous Catalysis, Vol. 2, Ch. 3.1 and 3.2, WileyVCH, Weinheim (1997).

[8] L. J. Boucher, A. A. Oswald, L. L. Murell, Prep., Div. Petrol Chem. 19, 162 (1974).

[9] B. Arkles, CHEMTECH 7, 766 (1977).

[10] J. P. Blitz, R. S. S. Murphy, D. E. Leyden, J. Colloid Interface Sci. 121, 63 (1988).

[11] C. P. Tripp, M. L. Hair, J. Phys. Chem. 97, 5693 (1993).

[12] P. Orth, H. Engelhardt, Chromatographia 15, 91 (1982).

[13] D. E. Leyden, Silanes, Surfaces and Interfaces, Gordon and Breach, New York (1985).

[14] H. Schumann, R. Widmaier, K.C. H. Lange, B.C. Wassermann, Z. Naturforsch. 60b, 614 (2005).

[15] a) W. Kaminsky, J. Kopf, H. Sinn, H. J. Vollmer, Angew. Chem. 88, 688 (1976); Angew. Chem. Int. Ed. Engl. 15, 629 (1976); b) A. Andresen, H. G. Cordes, J. Herwig, W. Kaminsky, A. Merck, R. Mottweiler, J. Pein, H. Sinn, H. J. Vollmer, Angew. Chem. 88, 689 (1976); Angew. Chem. Int. Ed. Engl. 15, 630 (1976); c) W. Kaminsky, in R. B. Seymour, T. Cheng (eds): History of Polyolefins, 257, Reidel Publ. Dordrecht (1986); d) H. Sinn, W. Kaminsky, H. J. Vollmer, R. Woldt, Angew. Chem. 92, 396 (1980); Angew. Chem. Int. Ed. Engl. 19, 390 (1980); e) W. Kaminsky, Macromol. Chem. Phys. 197, 3907 (1996). 